\title{
Article \\ Foot Insole Pressure Distribution during the Golf Swing in Professionals and Amateur Players
}

\author{
Enrique Navarro ${ }^{1, *+}{ }^{,}$, José M. Mancebo ${ }^{1,+}\left(\mathbb{D}\right.$, Sima Farazi $^{2}$, Malena del Olmo ${ }^{3}$ and David Luengo ${ }^{3}(\mathbb{D}$ \\ 1 Sport Biomechanics Laboratory, Health and Human Performance Department, Faculty of Physical Activity \\ and Sports Sciences-INEF, Universidad Politécnica de Madrid, 28040 Madrid, Spain; \\ jm.mancebo@alumnos.upm.es \\ 2 Continental AG, 30419 Hannover, Germany; sima.farazi@continental.com \\ 3 Audiovisual and Communications Engineering Department, Electrical Engineering School, Universidad \\ Politécnica de Madrid, 28040 Madrid, Spain; malena.delolmo.reillo@alumnos.upm.es (M.d.O.); \\ david.luengo@upm.es (D.L.) \\ * Correspondence: enrique.navarro@upm.es \\ + The first two authors contributed equally to this work.
}

check for updates

Citation: Navarro, E.; Mancebo, J.M.; Farazi, S.; del Olmo, M.; Luengo, D. Foot Insole Pressure Distribution during the Golf Swing in Professionals and Amateur Players Appl. Sci. 2022, 12, 358. https:// doi.org/10.3390/app12010358

Academic Editor: Claudio Belvedere

Received: 20 November 2021

Accepted: 23 December 2021

Published: 30 December 2021

Publisher's Note: MDPI stays neutral with regard to jurisdictional claims in published maps and institutional affiliations.

Copyright: (C) 2021 by the authors. Licensee MDPI, Basel, Switzerland. This article is an open access article distributed under the terms and conditions of the Creative Commons Attribution (CC BY) license (https:// creativecommons.org/licenses/by/ $4.0 /)$.

\begin{abstract}
There are numerous articles that study the ground reaction forces during the golf swing, among which only a few analyze the pressure pattern distributed on the entire surface of the foot. The current study compares the pressure patterns on the foot insoles of fifty-five golfers, from three different performance levels, playing swings with driver and 5-iron clubs in the driving range. Five swings were selected for each club. During each swing, ultra-thin insole sensors $\left(4\right.$ sensors $\left./ \mathrm{cm}^{2}\right)$ measure foot pressure at the frequency of $100 \mathrm{~Hz}$. To perform statistical analysis, insole sensors are clustered to form seven areas, with the normalized pressure of each area being our dependent variable. A video camera was used to label the five key instants of the swing. Statistical analysis demonstrates a significant difference between the pressure distribution pattern of the left and right feet for both driver and 5-iron. However, the pressure distribution pattern remains almost the same when switching the club type from 5-iron to driver. We have also observed that there are significant differences between the pattern of professionals and players with medium and high handicap. The obtained pattern agrees with the principle of weight transfer with a different behavior between the medial and lateral areas of the foot.
\end{abstract}

Keywords: golf swing; biomechanics; pressure patten; weight transfer; sensors

\section{Introduction}

The concept of weight transfer was first introduced as a popular term between players and coaches in an article in the Golf Digest magazine [1]. The weight transfer paradigm during the golf swing is a mechanical concept that golf coaches consider as an important metric for coaching the golf swing [2-4]. This concept has been intensively studied, mainly through variables obtained by means of a force platform, such as the distribution of the vertical force [5-9], the center of pressure [10-14], and the torque [7,9]. However, a lack of information exists about the weigh transfer based on the pressure measured between the foot and the shoe [15-17].

Ball and Best [12] has identified two styles of weight transfer based on the center of pressure (CoP) using cluster analysis: the "Front Foot" and the "Reverse Pivot" patterns. In the "Front Foot" pattern, players move the CoP backwards during the backswing and forward during the downswing phase. In the "Reverse Pivot" pattern, the CoP moves backwards during the backswing. However, after a short move forward, it moves again backwards and remains there during the downswing. On the other hand, Pataky [18], demonstrated that using only the center of pressure without considering the foot plantar distribution can be a limitation to properly understand the transmission of forces during 
the swing. Kawashima et al. [16], have analyzed the foot pressure distribution during the swing using an instrumented foot pressure insole. They used an insole with three pressure sensors to collect data from 14 players separated into two handicap groups. They compared players, but they did not report any specific statistical pattern of the foot pressure distribution. Worsfold et al. [19], analyzed the plantar pressure distribution in a sample of 18 players of different handicaps, but they compared only the maximum pressure without considering pressure variations during the whole swing.

Another important issue is the relation between the support forces, plantar pressures, and the clubhead speed as a way of assessing the player performance. Ball and Best [13], with a sample of different handicap players, indicated that a greater range and speed in the movement of the center of pressure during the downswing is associated with higher clubhead speeds in the "Front foot" pattern players. The same authors, Ref. [20], with a sample of five players, observed that a greater CoP displacement is associated with a greater clubhead speed $(p=0.05)$. Other authors have obtained a relation of the amount of reaction forces [8] and vertical torques [7,9] with the player's performance. Pataky [18], analyzed the relation between the plantar pressure and the clubhead speed. He measured golf swings of 32 players with handicaps between 2.7 and 25 and discovered that players with higher clubhead speeds also produce higher pressure values on the lateral side of the lead foot.

Several authors have studied the relation between foot pressures and club type. For instance, Refs. $[5,10,11]$ have analyzed the weight transfer of the golfers using different clubs, and they have proved that reaction forces are bigger when players use the driver instead of the iron club; however, the vertical torques are similar [21]. Ball and Best [14], concluded that the patterns of the $\mathrm{CoP}$ movement are similar when comparing golf swings with the driver and iron clubs. However, we have not found any information about the plantar pressures using different clubs.

Reaction forces, vertical torques [21], and insole foot pressures [15,19,22] have been also used to compare different shoes. Worsfold et al. [21], have analyzed the amount of vertical torque that exists between three types of shoes with different spikes and the natural grass surface in players of different levels. They observed that low handicap players generate more torque on the rear foot using the driver. The produced torque in metal spikes shoes is more significant than the flat-soled shoes. Ref. [19] also considered foot pressure insoles to compare metal spikes, alternative spikes, and flat shoes. Similarly, ref [22] developed a pressure map to assist in the development of a golf shoe. Indeed, it seems that most investigations about the foot plantar pressure belong to the design and development of golf shoes.

Our objective is to analyze the plantar pressure distributions in both feet during swings performed by players with different handicap levels using different clubs. Plantar pressure data are collected and statistically analyzed to discuss our proposed hypotheses: (1) the pressure data are different for the lead and rear foot; (2) the pressure data are different for different clubs; (3) the pressure data are different between players with different handicap levels; and (4) there exists a statistically significant and consistent pressure distribution pattern.

\section{Methods}

\subsection{Participants}

All the participants played golf more than once a week wearing the appropriate sport equipment and using their own clubs for our measurements. Subjects provided written informed consent and procedures were approved by the Ethics Committee of the Universidad Politécnica de Madrid (\#P2111600007). A total of 61 players were recorded, but 6 players were finally discarded for the data processing due to bad recording conditions. All subjects were right hand players. The fifty-five golf players were classified into three groups based on their level of performance applying the approach introduced by Choi et al. [8]: a group of professionals (Pro), a group of amateur players with a handicap 
equal to or less than 15 (Medium), and a group of amateur players with a handicap higher than 15 (High). These classes are named as Pro, Medium, and High in Table 1, and respectively contain 15,15 , and 25 players. Table 1 statistically describes height, age, and weight of players in each group by providing means and standard deviations. Driver and 5-iron club speeds at the impact time, expressed in miles per hour ( $\mathrm{mph}$ ), are also included in Table 1.

Table 1. Descriptive statistics of the sample (mean and standard deviation) for the three player groups considered: Pro, Medium, and High handicap. Driver (Mph) and 5-iron (Mph) refers to the club speed during the impact expressed in miles per hour.

\begin{tabular}{ccccccc}
\hline & Handicap & Height $(\mathbf{m})$ & Age (Years) & Weight $(\mathbf{k g})$ & Driver $(\mathbf{M p h})$ & 5-Iron $(\mathbf{M p h})$ \\
\hline Pro (15) & 0 & $1.76 \pm 0.05$ & $39 \pm 12.32$ & $81 \pm 12.65$ & $108.72 \pm 5.33$ & $92.81 \pm 4.18$ \\
Medium (15) & $9 \pm 4.82$ & $1.79 \pm 0.07$ & $48 \pm 14.71$ & $78.2 \pm 13.11$ & $103.44 \pm 7.17$ & $89.83 \pm 6.47$ \\
High (25) & $22.5 \pm 6.66$ & $1.78 \pm 0.05$ & $36.5 \pm 12.65$ & $77.95 \pm 10.17$ & $98.89 \pm 9.61$ & $85.85 \pm 8.12$ \\
\hline
\end{tabular}

\subsection{Data Collection}

The plantar pressures of each foot were recorded by means of two instrumented insoles (F-Scan; Tekscan model $3000 \mathrm{E}$ ) with a total of 900 pressure sensors working at $100 \mathrm{~Hz}$ [23]. To avoid any interference of the instrument with the player performance, the insoles were used under the original insoles of the golf shoe owned by each subject. Each insole is connected to the portable transmitter unit (Versatek VW-1) via a thin cable. The transmitter unit sends the information to the computer using a wireless protocol. In addition to the pressure sensor data, video images were recorded using a Sony A7 III video camera with a $20 \mathrm{~mm}$ fixed focal length lens working at $50 \mathrm{~Hz}$ and connected to the F-Scan system via HDMI port. Images and pressures data streams were synchronized internally by the system, and the key instants of the swing (stand (ST), middle backswing (MB), top of the backswing (TB), ball impact (IM), and early finish (EF)) were manually labeled. Figure 1 shows a graphical example of these five key instants. Simultaneously, we tracked the ball flight and the clubface movement via a launch monitor (Foresight model CG2 + HMMT), which consists of 4 stereoscopic high-speed cameras and detects small flat reflective markers ( $5 \mathrm{~mm}$ diameter) adhered to the ball and the club face. The launch monitor provides real time information to the players about their swing, including performance metrics such as the launch distance, the club speed, the ball speed, and the ball spin.

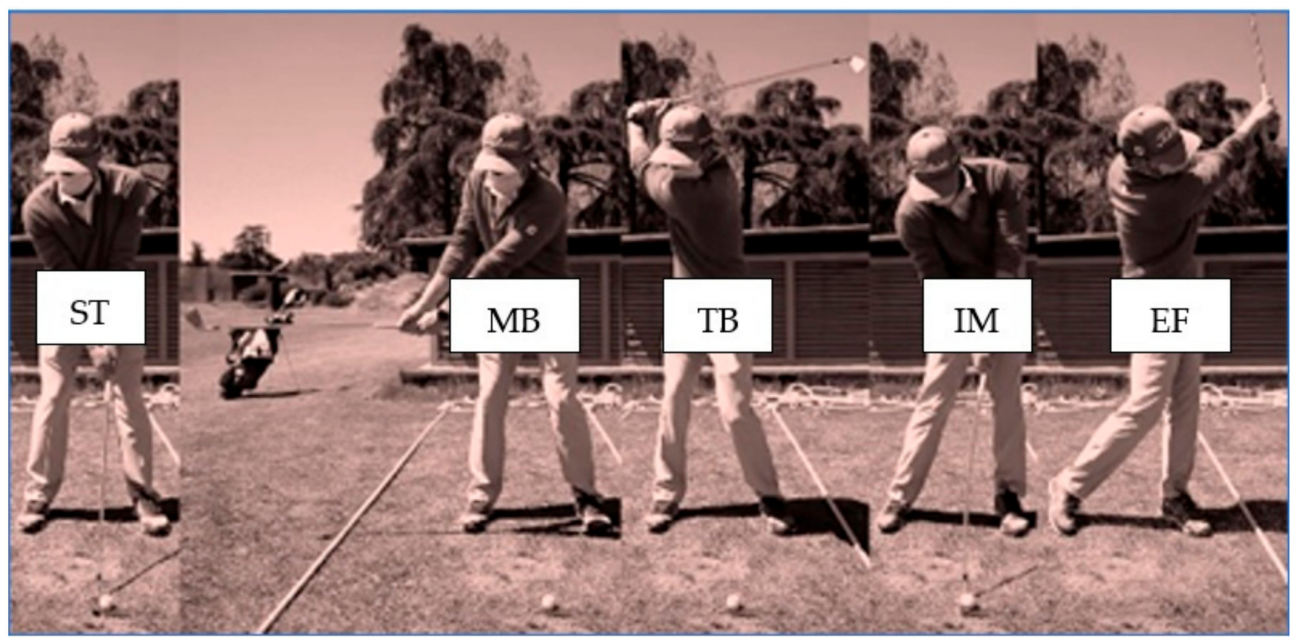

Figure 1. Key instants of the swing: Stand (ST), then the club start its movement; Middle Backswing (MB), when the club shaft is parallel to the ground; Top of backswing (TB), when the descent begins; Ball Impact (IM), when the club contacts the ball; Early finish (EF), when the saft surpasses the vertical axis to the ground. 
Each participant hit balls with the two clubs, until he made five good shots with each of the 5-iron and Driver club. After each shot, the player (under the supervision of a professional player) decides whether the shot was good enough or not using the information recorded by the launch monitor: ball release velocity, maximum flight, and distance of the trajectory. These five good shots for each club were chosen for the later analysis. Figure 2 shows an example of the recording environment.

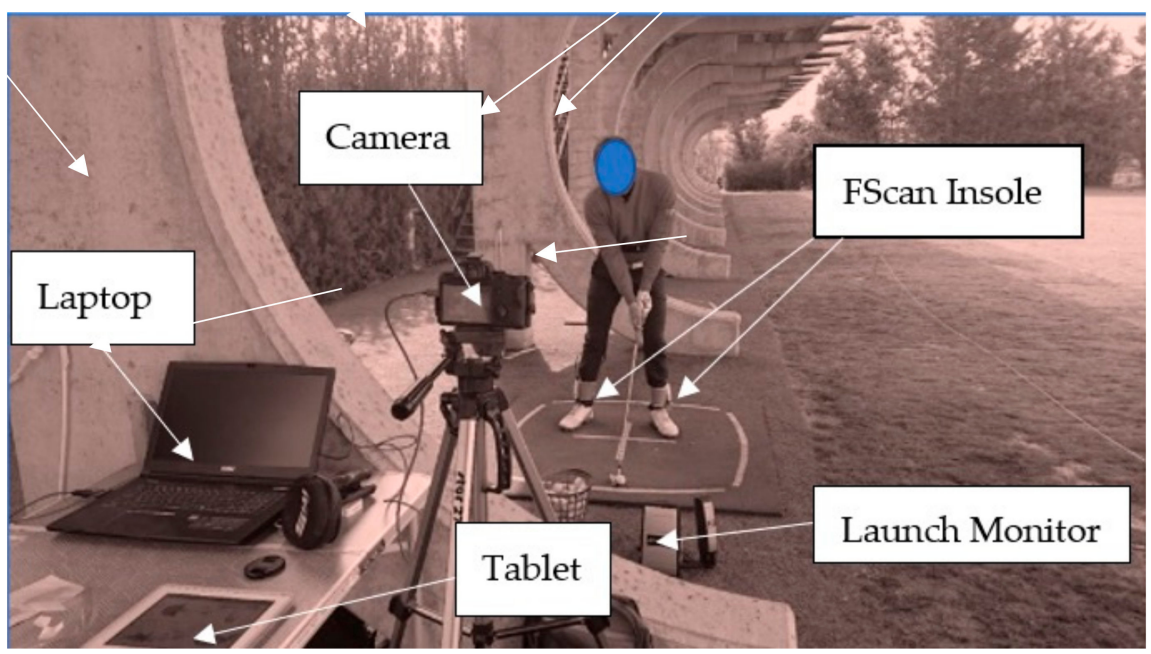

Figure 2. Data recording environment including the F-Scan insoles, the camera used to record the video sequences, the laptop and table which store the data, and the launch monitor used to assess the swing quality.

\subsection{Variables and Statistical Analysis}

The F-Scan software automatically detects the left and right insoles, capturing left and right footprints, respectively. For the statistical analysis, we have grouped the output from the 900 sensors of F-Scan into 7 anatomically relevant areas: toes (TOES), medial metatarsal (METmed), lateral metatarsal (METlat), plantar arc medial (ARCmed), plantar arc lateral (ARClat), heel medial (HEELmed), and heel lateral (HEELlat) (Figure 3). The pressure at each foot area is calculated by adding the recorded pressure (in Pascals) of all the sensors located in that area. Figure 4 presents an example of the pressure variation in time for each of these 7 areas, both for the left and the right foot, for a professional player performing a swing with the driver club.

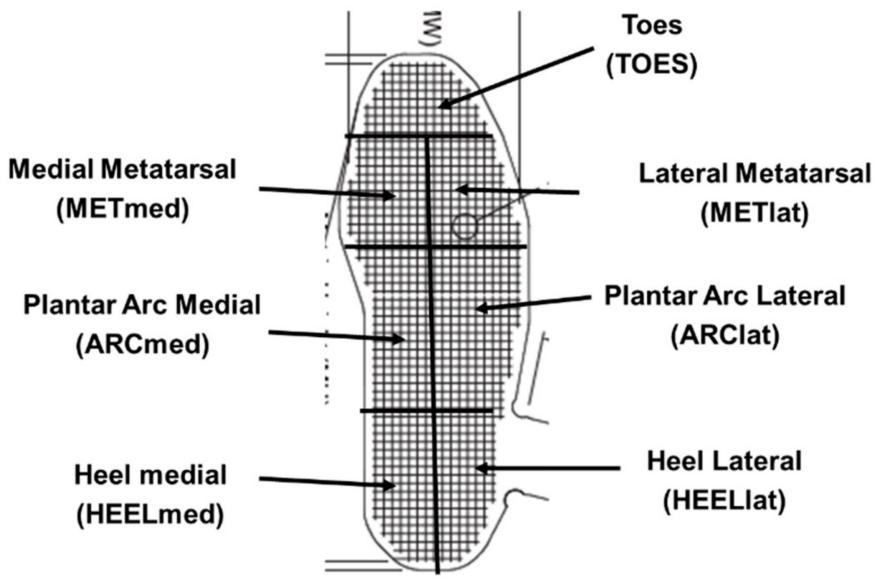

Figure 3. Seven Foot plantar areas defined for the statistical analysis: toes (TOES), medial metatarsal (METmed), lateral metatarsal (METlat), plantar arc medial (ARCmed), plantar arc lateral (ARClat), heel medial (HEELmed), and heel lateral (HEELlat). 

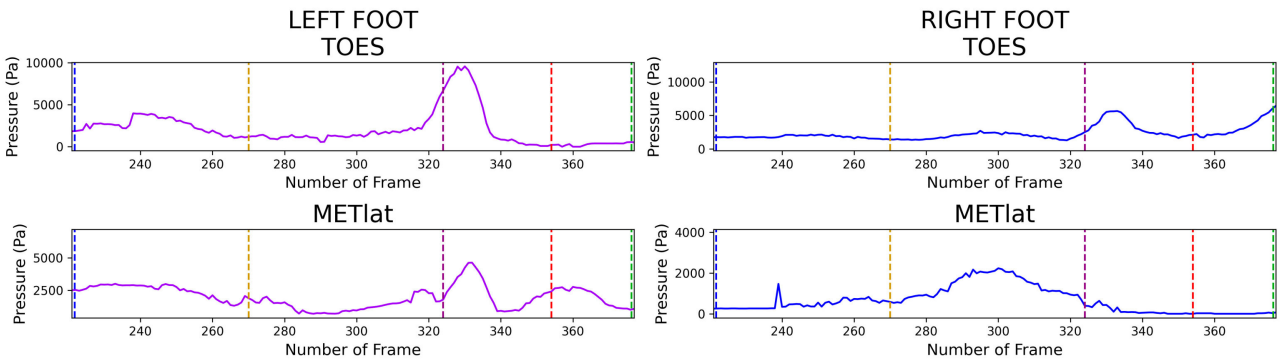

METmed

METmed
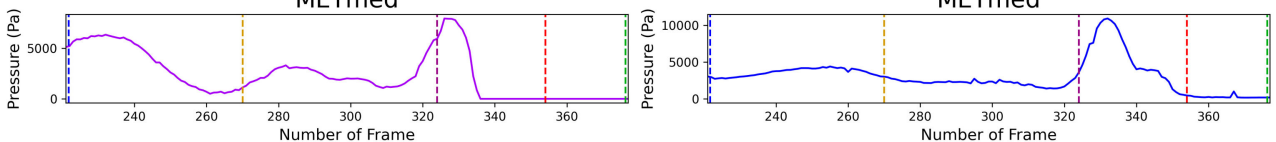

ARClat
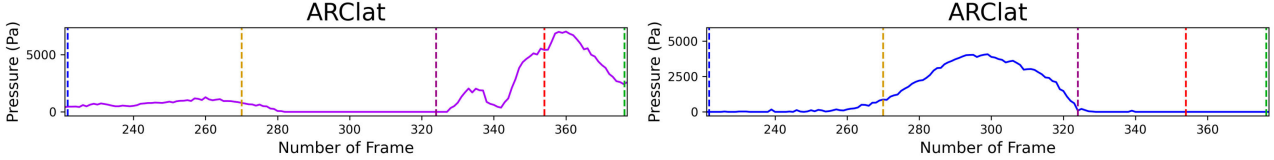

ARCmed

ARCmed
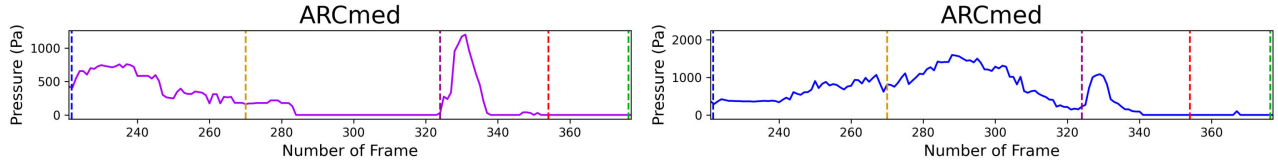

HEELlat

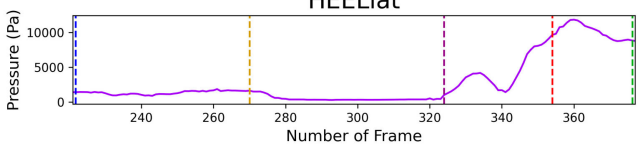

HEELlat
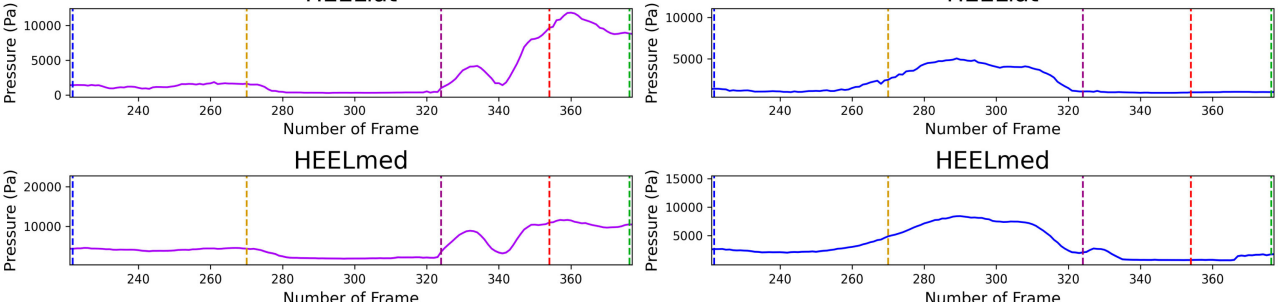

HEELmed

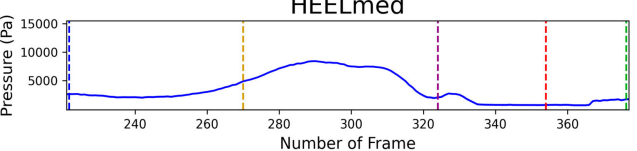

Figure 4. Pressure variation in time frames recorded for a professional player using a driver club in each of the 7 anatomical areas. Pressures plots on the left side belong to the left foot, whereas the pressure plots on the right side represents the right foot. Dashed lines show the key instants during the swing (blue: stand (ST), yellow: middle back swing (MB), purple: top back swing (TB), red: impact (IM), and green: finish (EF)).

The dependent variable $P \%_{j}$ is the normalized pressure, which is defined as the ratio of the absolute pressure $P_{j}$ in the $j$ th area divided by the total pressure in the foot (summation of the pressures of all areas) in percentage:

$$
P \%_{j}=\frac{P_{j}}{\sum_{i=1}^{7} P_{i}}
$$

Five independent variables were used:

1. Players handicap levels (Pro, Medium and High) as indicated in Table 1.

2. Clubs:
a. 5-Iron.
b. Driver.

1. Foot sides:
c. Right (rear)
d. Left (lead)

1. $\quad$ Areas: The 7 areas defined in Figure 3.

2. Time (Key instants): The 5 moments shown in Figure 1.

Average values of the 5 repeated trials were used in the statistical analysis following Han et al. [9]. The ANOVA test, with Groups (3) $\times$ Clubs (2) as inter subjects variables 
and Areas (7) $\times$ Time (5) as repeated variables, was used separately for each foot. The Leveune's test was previously applied to check the homogeneity of the variance. The effect size was measured using omega squared (0.01 is small, 0.06 is medium, and 0.14 is large). After studying the main effects, a pairwise comparison of the variables was carried out, correcting the Type II error using the Bonferroni adjustment (Thomas and Nelson, 2001).

\section{Results}

The Foot Sides variable significantly affects $P \%$, the normalized pressure of each area during the swing, with a large effect size $\left(F_{24.157}=51.089, p=0.001, \eta 2=0.886\right)$. This means that the pattern of foot pressure distribution is significantly different for the right (rear for right-handed player) and left foot (lead for right-handed player). The 35 pair comparisons between left and right foot sides of the 7 areas during the 5 key instants in each foot for the professional players were made for each club. Among these 70 comparisons, 54 cases revealed significant differences. Figure 5 shows the normalized pressures for the five areas (TOES, METlat, METmed, ARClat, and HEELmed) where statistically significant differences were found in one or more key time instants (these cases are marked with * symbols). The remaining two areas (ARCmed and HEELlat) are not shown because no statistically significant differences were found at any of the key time instants.
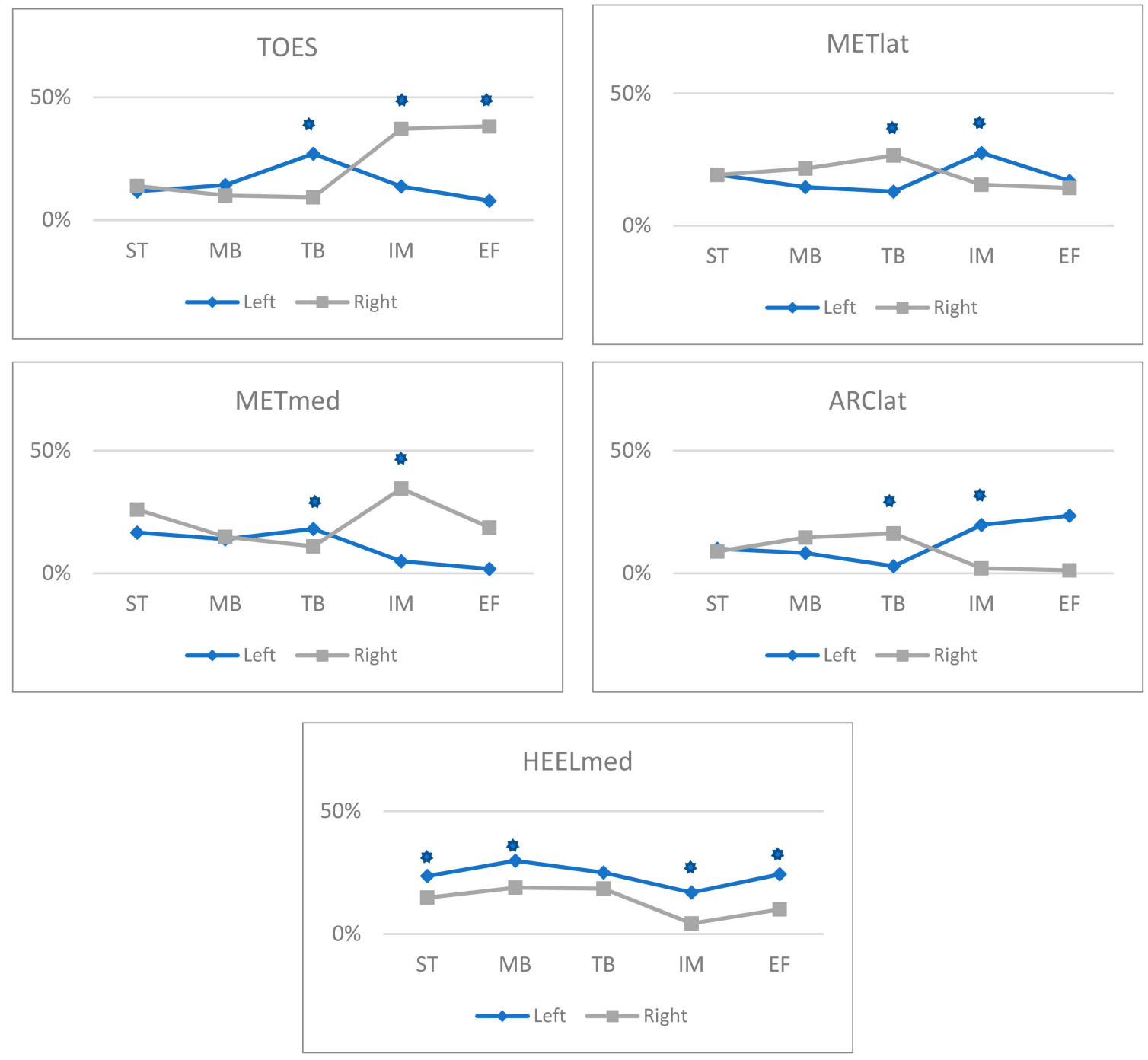

Figure 5. Comparison of the normalized pressure for the Professional Players during the swing in the left and right foot. ( $\left.{ }^{*}\right)$ : Significant difference $(p<0.05)$. 
Regarding the two analyzed clubs, it was found that the pressures in the different areas of the insole of the foot throughout the swing are not significantly different from a statistical point of view between the driver and the 5 -iron for both the left $\left(F_{24,67}=0.938, \rho=0.553\right.$, $(\eta 2=0.938)$ and right foot $\left(F_{24.67}=1.107, p=0.360, \eta 2=0.284\right)$.

On the other hand, it was found that the pressures of foot insole areas are significantly different, from a statistical point of view, through the swing for the different groups of players regardless of the club they use for both the left foot $\left(F_{48.136}=1.863, p=0.003, \eta 2=0.398\right)$ and the right foot $\left(F_{48.136}=1.831, p=0.004, \eta 2=0.399\right)$. On the Tables 2 and 3 are presented the differences in the normalized pressure between the professionals and the high handicap players and between the professionals and the medium handicap for both right (rear) and left (lead) foot.

Table 2. Differences in the means of the normalized pressures in the left foot (lead) between Professionals and High handicap players (Difference Pro-High) and Professionals versus Medium handicap players (Difference Pro-Medium) at the key time instants (ST (Stand), MB (middle backswing), TB (top backswing), IM (Impact), EF (early finish)) in the seven previously defined areas (Toes (all toes), METlat (lateral metatarsal), METmed (medial metatarsal), ARClat (lateral arch), ARCmed (medial arch) HEELlat (lateral heel), HEELmed (medial heel)). The * denotes a statistically significant difference between the compared groups (Professional vs. high or Professional vs. Medium) with significance level $p<0.05$.

\begin{tabular}{|c|c|c|c|c|c|c|c|c|c|c|c|}
\hline & \multirow[b]{2}{*}{ Areas } & \multicolumn{2}{|c|}{ ST } & \multicolumn{2}{|c|}{ MB } & \multicolumn{2}{|c|}{ TB } & \multicolumn{2}{|c|}{ IM } & \multicolumn{2}{|c|}{ EF } \\
\hline & & Mean & $p$ & Mean & $p$ & Mean & $p$ & Mean & $p$ & Mean & $p$ \\
\hline \multirow{7}{*}{$\begin{array}{l}\text { Difference } \\
\text { Pro-High }\end{array}$} & TOES & $5 \%(*)$ & $p<0.05$ & $5 \%$ & $p<0.05$ & \multirow{7}{*}{$\begin{array}{c}6 \% \\
0 \% \\
-\mathbf{1 0} \% \\
\left(^{*}\right) \\
-2 \% \\
0 \% \\
2 \% \\
3 \% \\
\end{array}$} & $p<0.05$ & $-4 \%$ & $p<0.05$ & $1 \%$ & $p<0.05$ \\
\hline & METlat & $2 \%$ & $p>0.05$ & $0 \%$ & $p>0.05$ & & $p>0.05$ & $-4 \%$ & $p>0.05$ & $-12 \%\left(^{*}\right)$ & $p<0.001$ \\
\hline & METmed & $3 \%$ & $p>0.05$ & $-5 \%$ & $p>0.05$ & & $p<0.05$ & $-7 \%$ & $p>0.05$ & $-2 \%$ & $p>0.05$ \\
\hline & ARClat & $-3 \%$ & $p>0.05$ & $-2 \%$ & $p>0.05$ & & $p>0.05$ & $4 \%$ & $p>0.05$ & $-1 \%$ & $p>0.05$ \\
\hline & ARCmed & $2 \%$ & $p>0.05$ & $1 \%$ & $p>0.05$ & & $p>0.05$ & $0 \%$ & $p>0.05$ & $0 \%$ & $p>0.05$ \\
\hline & HEELlat & $-4 \%$ & $p>0.05$ & $-1 \%$ & $p>0.05$ & & $p>0.05$ & $8 \%\left(^{*}\right)$ & $p<0.05$ & $11 \%\left(^{*}\right)$ & $p<0.05$ \\
\hline & HEELmed & $-5 \%$ & $p>0.05$ & $2 \%$ & $p>0.05$ & & $p>0.05$ & $4 \%$ & $p>0.05$ & $3 \%$ & $p>0.05$ \\
\hline \multirow{7}{*}{$\begin{array}{c}\text { Difference } \\
\text { Pro-Medium }\end{array}$} & TOES & $6 \%\left(^{*}\right)$ & $p<0.05$ & $7 \%\left(^{*}\right)$ & $p<0.05$ & $7 \%$ & $p>0.05$ & $2 \%$ & $p>0.05$ & $1 \%$ & $p>0.05$ \\
\hline & METlat & $3 \%$ & $p>0.05$ & $2 \%$ & $p>0.05$ & $0 \%$ & $p>0.05$ & $-3 \%$ & $p>0.05$ & $-8 \%\left(^{*}\right)$ & $p<0.05$ \\
\hline & METmed & $-1 \%$ & $p>0.05$ & $-5 \%$ & $p>0.05$ & $-8 \%$ & $p>0.05$ & $-6 \%$ & $p>0.05$ & $-2 \%$ & $p>0.05$ \\
\hline & ARClat & $0 \%$ & $p>0.05$ & $-1 \%$ & $p>0.05$ & $-2 \%$ & $p>0.05$ & $5 \%$ & $p>0.05$ & $1 \%$ & $p>0.05$ \\
\hline & ARCmed & $1 \%$ & $p>0.05$ & $1 \%$ & $p>0.05$ & $-2 \%$ & $p>0.05$ & $0 \%$ & $p>0.05$ & $0 \%$ & $p>0.05$ \\
\hline & HEELlat & $-5 \%$ & $p>0.05$ & $-4 \%$ & $p>0.05$ & $-1 \%$ & $p>0.05$ & $0 \%$ & $p>0.05$ & $3 \%$ & $p>0.05$ \\
\hline & HEELmed & $-4 \%$ & $p>0.05$ & $0 \%$ & $p>0.05$ & $6 \%$ & $p>0.05$ & $2 \%$ & $p>0.05$ & $5 \%$ & $p>0.05$ \\
\hline
\end{tabular}

Table 3. Differences in the means of the normalized pressures in the right foot (rear) between Professionals and High handicap players (Difference Pro-High) and Professionals versus Medium handicap players (Difference Pro-Medium) at the key time instants (ST (Stand), MB (middle backswing), TB (top backswing), IM (Impact), EF (early finish)) in the seven previously defined areas (Toes (all toes), METlat (lateral metatarsal), METmed (medial metatarsal), ARClat (lateral arch), ARCmed (medial arch) HEELlat (lateral heel), HEELmed (medial heel)). The * denotes a statistically significant difference between the compared groups (Professional vs. high or Professional vs. Medium) with significance level $p<0.05$.

\begin{tabular}{|c|c|c|c|c|c|c|c|c|c|c|c|}
\hline & \multirow[b]{2}{*}{ Areas } & \multicolumn{2}{|c|}{ ST } & \multicolumn{2}{|c|}{ MB } & \multicolumn{2}{|c|}{ TB } & \multicolumn{2}{|c|}{$\mathrm{IM}^{*}$} & \multicolumn{2}{|c|}{$\mathrm{EF}$} \\
\hline & & Mean & $p$ & Mean & $p$ & Mean & $p$ & Mean & $p$ & Mean & $p$ \\
\hline \multirow{7}{*}{$\begin{array}{l}\text { Difference } \\
\text { Pro-High }\end{array}$} & TOES & $5 \%(*)$ & $p<0.01$ & $1 \%$ & $p<0.05$ & $0 \%$ & $p<0.05$ & $3 \%$ & $p<0.05$ & $-7 \%$ & $p<0.05$ \\
\hline & METlat & $2 \%$ & $p>0.05$ & $4 \%$ & $p>0.05$ & $3 \%$ & $p>0.05$ & $-1 \%$ & $p>0.05$ & $8 \%\left(^{*}\right)$ & $p<0.05$ \\
\hline & METmed & $10 \%\left(^{*}\right)$ & $p<0.01$ & $2 \%$ & $p>0.05$ & $2 \%$ & $p<0.05$ & $-2 \%$ & $p>0.05$ & $1 \%$ & $p>0.05$ \\
\hline & ARClat & $-1 \%$ & $p>0.05$ & $3 \%$ & $p>0.05$ & $-1 \%$ & $p>0.05$ & $-1 \%$ & $p>0.05$ & $0 \%$ & $p>0.05$ \\
\hline & ARCmed & $1 \%$ & $p>0.05$ & $0 \%$ & $p>0.05$ & $0 \%$ & $p>0.05$ & $-1 \%$ & $p>0.05$ & $-1 \%$ & $p>0.05$ \\
\hline & HEELlat & $-7 \%\left(^{*}\right)$ & $p<0.05$ & $-5 \%$ & $p>0.05$ & $-2 \%$ & $p>0.05$ & $2 \%$ & $p<0.05$ & $2 \%$ & $p<0.05$ \\
\hline & HEELmed & $-9 \%\left(^{*}\right)$ & $p<0.05$ & $-5 \%$ & $p>0.05$ & $-1 \%$ & $p>0.05$ & $0 \%$ & $p>0.05$ & $-4 \%$ & $p>0.05$ \\
\hline
\end{tabular}


Table 3. Cont.

\begin{tabular}{|c|c|c|c|c|c|c|c|c|c|c|c|}
\hline & \multirow[b]{2}{*}{ Areas } & \multicolumn{2}{|c|}{ ST } & \multicolumn{2}{|c|}{ MB } & \multicolumn{2}{|c|}{ TB } & \multicolumn{2}{|c|}{$\mathrm{IM}^{*}$} & \multicolumn{2}{|c|}{ EF } \\
\hline & & Mean & $p$ & Mean & $p$ & Mean & $p$ & Mean & $p$ & Mean & $p$ \\
\hline \multirow{7}{*}{$\begin{array}{c}\text { Difference } \\
\text { Pro-Medium }\end{array}$} & TOES & $4 \%$ & $p<0.05$ & $0 \%$ & $p<0.05$ & $-3 \%$ & $p>0.05$ & $4 \%$ & $p>0.05$ & $4 \%$ & $p>0.05$ \\
\hline & METlat & $7 \%\left(^{*}\right)$ & $p<0.05$ & $5 \%$ & $p>0.05$ & $1 \%$ & $p>0.05$ & $5 \%$ & $p>0.05$ & $8 \%(*)$ & $p<0.05$ \\
\hline & METmed & $10 \%\left(^{*}\right)$ & $p<0.01$ & $2 \%$ & $p>0.05$ & $-1 \%$ & $p>0.05$ & $-1 \%$ & $p>0.05$ & $2 \%$ & $p>0.05$ \\
\hline & ARClat & $-1 \%$ & $p>0.05$ & $2 \%$ & $p>0.05$ & $1 \%$ & $p>0.05$ & $1 \%$ & $p>0.05$ & $1 \%$ & $p>0.05$ \\
\hline & ARCmed & $0 \%$ & $p>0.05$ & $0 \%$ & $p>0.05$ & $-1 \%$ & $p>0.05$ & $-1 \%$ & $p>0.05$ & $-2 \%$ & $p>0.05$ \\
\hline & HEELlat & $-8 \%\left(^{*}\right)$ & $p<0.05$ & $-5 \%$ & $p>0.05$ & $0 \%$ & $p>0.05$ & $-3 \%$ & $p>0.05$ & $-3 \%$ & $p>0.05$ \\
\hline & HEELmed & $-10 \%\left(^{*}\right)$ & $p<0.01$ & $-3 \%$ & $p>0.05$ & $3 \%$ & $p>0.05$ & $-5 \%$ & $p>0.05$ & $-9 \%\left(^{*}\right)$ & $p<0.05$ \\
\hline
\end{tabular}

As Figure 5 shows, it was found that the normalized pressure in the different areas of the insole of the foot is significantly different between the different instants of the swing, both for the lead (left) foot $\left(F_{24.67}=0.994, p=0.000, \eta 2=0.994\right)$ and for the rear (right) foot $\left(F_{24.67}=0.945, \quad p=0.000, \eta 2=0.945\right)$ regardless of the analyzed player group.

\section{Discussion}

The pressure data from sensors between the foot and the shoe were analyzed during the golf swing using an instrumented insole. We consider pressure of the seven anatomical areas normalized with respect to the total pressure of each foot. It is known that the swing performance depends primarily on the velocity vector and the orientation of the clubface at the ball contact, as well as the forces applied during the impact. To perform a good swing, players move their body parts sequentially, starting with lower limbs, which are furthest from club head to move the club in a plane during downswing [24]. Hence, the player must generate the necessary reaction forces between his feet and the ground during the whole swing [9]. Four hypotheses with respect to the plantar pressure during the swing were formulated at the beginning of this work. In the following, we discuss these hypotheses in detail regarding our obtained results and statistical analysis.

Hypothesis \# 1 was confirmed. The pressure distribution pattern of the rear foot (right in right-handed player) is significantly different from that of the lead foot (left in right-handed player).

The pair comparisons of the pressure in the seven anatomical areas between the left and the right feet throughout the swing show significant differences in 54 out of the 70 cases (Figure 5). At the top of the backswing, in the lateral parts of the metatarsal (METlat), arch (ARClat), and heel (HEELlat), we have observed that pressure reaches a maximum on the right foot, whereas the same areas show a minimum on the left foot. However, pressure patterns at the lateral areas (METlat, ARClat, and HEELlat) inversely evolve during the downswing, the phase between top of backswing and impact instants. During the downswing phase, the pressure at the right foot decreases in these three lateral areas, while it increases at the left foot, reaching their maximum at the impact (Figure 5). Interestingly, in comparison with the lateral regions, our results show a reverse behavior in the toes and medial metatarsals (TOES and METmed) areas. Figure 5 shows that in TOES and METmed areas, pressure reaches a maximum during the backswing in the left foot (lead), while in the right foot (rear), pressure maximum occurs during the downswing. In the medial arch area (ARCmed), we observed insignificant differences between pressures of the left and the right feet. In both feet, medial arch areas (ARCmed) show the same pattern of pressure distribution, contributing to less than $8 \%$ of the total pressure. In the medial part of the heel (HEELmed), both feet behave also in a similar way, albeit the contribution of this area to the total pressure is higher in the left foot. In addition, HEELmed areas of both feet reach a maximum at the middle backswing instant and a minimum at the impact moment.

The former results agree with those obtained by the other authors who have analyzed the plantar pressures $[15,16,18,19]$. According to [15] the loads are different for each foot, so that greater pressure is produced first on the rear foot before the top of the swing, and then on the lead foot just before impact. The maximum pressures are different in each foot according to [18], occurring in the lateral forefoot, heel, and hallux of the leading foot and 
in the medial forefoot and hallux of the rear. Worsfold et al. [19], have also found that the behavior of the feet is different in relation with the insole pressures, showing a very similar information to ours, but without presenting statistical results.

The most relevant conclusion of the former results is that plantar pressures agree with the classic principle of "weight transfer" from the rear foot to the lead [25]. This transfer in weight has been studied through reaction forces [6-9], through the movement of the center of pressure in each foot [10,11], or through the location of the total center of pressure [12-14]. In all cases, they argued the existence of a movement of the forces and the center of pressure towards the rear foot during the backswing, followed by a transfer towards the lead foot during the downswing. It should be mentioned that this is the so called "standard" pattern, but according to some authors there are players who opt for a so-called "reverse" pattern that shifts the weight towards the rear foot during the impact $[12,13]$. However, our results only partially agree with the classic concept of weight transfer because we have shown that the transmission of loads is different and depends on the specific foot plantar area. We found out that in the lateral part of the foot (METlat, ARClat, and HEELlat), the right foot is loaded before the left foot, while in the medial metatarsus and toes (METmed and TOES) the weight shift occurs in a reverse pattern, and the medial arch and medial heel (ARCmed and HEELmed) do not reveal any weight shifting between right and left feet. Further studies are needed to investigate whether this pressure transfer pattern (Figure 5) is unique or there are alternative patterns.

Hypothesis \# 2 is discarded. The pressures distributions are not different for the driver and the 5-iron.

It is very important that this hypothesis is investigated for a proper swing training, since it means that the insole pressure distribution pattern in the swing is the same regardless of the applied club. Several authors have studied the foot pressure during the golf swing for a single club $[15,16,18,19]$, but we have not found any article comparing the foot pressure distribution using different clubs. Authors that used ground reaction forces to compare different clubs have shown that the generated forces by the driver $(703 \mathrm{~N})$ are higher than those produced by the 5-iron ( $695 \mathrm{~N}$ ) [5], while the 3-iron and the 7-iron clubs have similar force values $[10,11]$. From our point of view, it is an expected result that the absolute forces using the driver are higher than those using the irons, because the driver has higher moment of inertia (MOI), and thus requires more force to be moved. In our case, we have not observed differences between clubs, since the pressure has been normalized with respect to the total foot pressure, hence avoiding the effects of the club types and weight of players. In addition, other authors have recorded similar vertical torques in the driver than in the 3-iron and the 5-iron [21], as well as similar movement of the CoP using the driver, the 3-iron or the 5-iron [14]. Therefore, our results seem to agree with the literature when the torques or the center of pressure are analyzed. However, further studies are needed to see whether the pressure pattern is similar for any club, including the putter.

Hypothesis \# 4 was confirmed. There exists a statistically significant and consistent pressure distribution pattern when players are analyzed all together. However, when the pattern is compared separately, the differences between the three handicap groups are also statistically significant, thus confirming Hypothesis \# 3.

\subsection{Stand-Right Foot}

Standing on the right foot during the starting position (Figure 5), we have found out that the highest pressure appears on the metatarsals; being higher in the medial metatarsal (METmed) area than in the lateral one (METlat); although, this difference is not statistically significant. The toes, the lateral arch, and the heels (TOES, ARClat, HEELmed, and HEELlat) have lower and similar pressures ( $9 \%$ to $14 \%$ ), while the medial arch pressure (ARCmed) is significantly the lowest. Worsfold et al. [19], analyzed 19 players of different handicap levels using the driver, and reported a similar pressure distribution; although, they only described the pressures qualitatively - without the specific pressure values. Indeed, there are controversial discussions regarding the location of the center of pressure in the stand 
moment on the rear foot. There are authors who place the CoP in a centered position on the right foot, which coincides with our results [10], while other studies place the CoP near the heels [11]. However, it is worthwhile mentioning that accurately estimation of the pressure distribution from the CoP location is impossible, since different pressure configurations might lead to identical positions of the CoP [18].

By standing on the right (rear) foot, we have found the highest number of statistically significant differences between players. As shown in Table 3, in comparison with the higher handicap players, professional players have higher pressure in the toes (Dif $=+5.5 \%, p=0.015$ ) and in the medial metatarsals (Dif $=+10.0 \%, p=0.003$ ) and lower pressure in the medial heel (Dif $=-9.5 \%, p=0.011$ ) and lateral heel (Dif $=-7.1 \%, p=0.03$ ). The observation is similar when comparing medium handicap players with professionals, with the exception that statistically significant differences are found in the lateral metatarsals (METlat) and not in the toes. These results suggest that amateur players should place higher pressure on the forefoot of the rear foot, both in medial and lateral metatarsals (METmed and METlat) during the stand instant.

\subsection{Stand-Left Foot}

Figure 5 shows that at the starting position, on the leading foot of the professionals, the highest pressure belongs to the heel areas, with the pressure in the medial part of the heel (HEELmed) being significantly higher than at the lateral part (HEELlat), and in the metatarsals (similar pressure in METmed and METlat). The second level of pressure was found in the toes and the lateral arch (TOES and ARClat), which have similar pressures. The lowest pressure occurs in the medial arch (ARCmed); although, its difference with the lateral arch (ARClat) is not significant. This pressure distribution coincides with the one found by Worsfold et al. [19], who used a similar methodology to the one in the current study. This pressure distribution is also consistent with that found by other authors who analyzed the center of pressure on each foot $[10,11]$.

In comparison with the medium and high handicap groups, Table 2 shows that professionals have higher pressure on the toes of the leading foot (Dif $=+4.9 \%, p=0.035$ ). We can recommend the players to push the ground with their leading foot, distributing the pressure evenly with a tendency of greater pressure on the heels, while not forgetting to properly contact the toes against the ground. However, further experimental research should be performed in the future to justify these recommendations.

\subsection{Middle Backswing-Right Foot}

At the middle backswing, we have observed that the pressure at the rear foot is almost evenly distributed between metatarsals, heels, and the lateral arch (METmed, METlat, HEELmed, HEELlat, and ARClat), with the highest pressure recorded in the lateral metatarsal (METlat); although, the differences are not statistically significant (Figure 5). The lowest pressure levels are in the toes and in the medial arch (ARCmed). These results are in agreement with those obtained by Worsfold et al. [19]. Interestingly, it can be observed in Table 3 that the pressures of the professional are higher at the forefoot and lower at the heels, but these differences are not significant.

\subsection{Middle Backswing-Left Foot}

At the middle backswing, in the front foot Figure 5 shows that the highest pressure appears at the medial part of the heels (HEELmed), while the second level of pressure is at the toes. Metatarsals (METmed and METlat), lateral arch (ARClat), and lateral heel (HEELlat), reveal similar pressures. This result agrees with the one obtained by other authors who also used foot insole pressure [16,19]. It is remarkable that the pressure measured at the medial heel (HEELmed) corresponds to the maximum pressure recorded during the swing in the left foot. This coincides with [18], who studied plantar pressures using similar measurement tool. 


\subsection{Top Backswing-Right Foot}

On the rear foot at the top position of the backswing, the pressure is distributed in a similar way as in the middle backswing: the pressure is almost evenly distributed between metatarsals, heels, and the lateral arch (METmed, METlat, HEELmed, HEELlat, and ARClat), with the highest pressure at the lateral metatarsal (METlat). In this case, it is important to remark that there is quite a controversy regarding the distribution of plantar pressures at the top of the backswing on the rear foot. Kawashima et al., [16], used an instrumented insole with three sensors and analyzed 14 players without professionals), found that during the backswing the players with the lowest handicap had higher pressure on the lateral metatarsal, which agrees with our results. However, using 8 piezoelectric pressure sensors in players with handicaps between 5 and 10, Wallace et al. [15], reported that maximum pressure on the rear foot occurred before the top backswing on the heel, styloid process, and metatarsals 4 and 5, but they did not specify exactly where the highest pressure was exactly located. Other authors place the greatest pressure on the heels also [19] or allocate the center of pressure towards the heels on the right (i.e., rear) foot [10,11]. From our results, we conclude that on the rear foot at the top of the backswing, the pressure is distributed between the anterior and posterior parts with a statistically significant higher pressure on the lateral areas (METlat and ARClat) of the foot.

\subsection{Top Backswing - Left Foot}

At the top of the backswing on the left foot, we have found that the highest pressure occurs in the toes, medial metatarsal (METmed), and medial heel (HEELmed), with the differences between them not being statistically significant. The second level of pressure corresponds to the lateral metatarsal (METlat) and the lateral heel (HEELlat). The lowest significant pressure occurs at the lateral and medial arches (ARClat and ARCmed), with less than $3 \%$ of the total pressure. These data coincide with Worsfold et al. [19], who also found that the highest pressure appearing in the medial metatarsal. Again, it is very interesting to consider the difference between the information obtained from the center of pressure and the pressure distribution in the insole. Williams et al. [10] and Koenig et al. [11] report that the center of pressure moves forward during the backswing, reaching the most advanced position in the medial metatarsus of the lead foot at the top of the backswing. However, this information can be confusing for players and coaches because, according to our results and those of Worsfold et al. [19], the pressure on the medial heel (HEELmed) is similar to that on the medial metatarsal (METmed). Furthermore, it has been found that lower handicap players have higher pressure on the heel of the front foot during the backswing than players with higher handicap [16].

When comparing the groups of players at the top backswing for the lead foot, we have found a statistically significant difference in the medial metatarsal (METmed), indicating that the professionals have less pressure (Dif $=-9.6 \%, p=0.016$ ) than the players with higher handicap (Table 2). This can be interpreted as follows: professionals do not concentrate all the pressure on the medial metatarsal at the top backswing, shifting some pressure to the heels, possibly to anticipate the transmission of the pressure to the lateral part of the foot during the downswing.

\subsection{Impact-Right Foot}

During the downswing, the pressure on the rear foot moves towards the toes and the medial metatarsus (METmed), being these two pressures significantly higher than those at the rest of the areas at the impact, which agrees with the literature $[10,11,15,18,19]$. Interestingly, among all areas, the pressure on the toes is the maximum pressure recorded during the swing in the right foot. This agrees with Pataky, [18], who claims that the pressure on the distal part of the rear foot is positively correlated with the club speed, which reaches its maximum value at the impact. We have not found any statistically significant differences on the pressures between players in the three handicap groups; although, [16] have reported that lower handicap players showed less pressure at the 
lateral metatarsal and heel (METlat, HEELmed, and HEELlat) of the rear foot. Possibly, the sampling frequency $(100 \mathrm{~Hz})$ that we used was insufficient to find significant differences between professionals and amateurs due to the short duration of the downswing.

\subsection{Impact-Left Foot}

The highest pressure at the impact in the left foot is distributed between the lateral metatarsal, lateral arch, and heel (METlat, ARClat, HEELlat, and HEELmed), with higher pressure in the lateral metatarsal (METlat); although, the difference with the previous ones is not statistically significant. In the second level of pressure, we have the toes, and in the third place the medial metatarsal (METmed) and the medial arch (ARCmed), being these differences statistically significant. These results coincide with those found by other authors $[10,11,15,18,19]$. We have also observed (Table 2) that professional players have higher pressure in the lateral heel (HEELlat) on the lead foot (Dif $=7.8 \% ; p=0.017$ ), indicating the importance of applying pressure on both the lateral forefoot and heel during the impact. Pataky, [18], also using the F-Scan pressure insoles in a sample of amateur players of different handicaps, found that pressure on the lateral part of the front foot was positively correlated with club speed.

\subsection{Early Finish-Right Foot}

At the early finish, the highest pressure in the right foot is transferred mainly to the toes and, and in a second level, to the metatarsals and heel (METmed, METlat, HEELmed, and HEELlat). Although the pressure on the medial metatarsal (METmed) is higher than on the lateral (METlat), these differences are not statistically significant. The pressures in the lateral part of the heel (HEELlat) are greater than in the medial part (HEELmed), but the differences are statistically insignificant. These results agree with Williams et al. [10], Kawashima et al., [16], and Worsfold et al. [19]. Comparing the data between the different handicap levels (Table 3), we have found that the professionals have higher pressure on the lateral metatarsal (METlat) of the right foot ( $\mathrm{Dif}=+8.3 \% ; p=0.014$ ) than players with higher handicap. This finding indicates that professionals delay the raising of the heel during the follow through, pressing more than amateur players with the lateral part of the rear foot during the early finish [16]. Therefore, to avoid the early lift of the right heel, armatures players could push more on the medial part of the metatarsals and heels.

\subsection{Early Finish-Left Foot}

At the early finish, the highest pressure in the left foot occurs in the heel, both on the medial (HEELmed) and lateral areas (HEELlat), closely followed by the lateral arch (ARClat), and then by the lateral metatarsal (METlat); although, the difference is not statistically significant. The rest of the pressure is found in the toes and the medial part of the metatarsal and plantar arch (METmed and ARCmed). Once more, this coincides with the results obtained by other authors $[10,16,19]$. After the impact, Table 2 shows that professionals have less pressure on the lateral metatarsal (Dif $=-11.5 \%, p=0.001$ ) and more pressure on the lateral heel ( $\mathrm{Dif}=+11.1 \%, p=0.012$ ) than the players with the highest handicap at the early finish, indicating a transmission of the pressure to the heel of the lead foot. This is partially in agreement with the literature, since authors such as [16] found that professionals have higher pressure on the lateral heel (HEELlat), but also on the lateral metatarsal (METlat), than players with a lower level of performance. Unfortunately, these authors have not precisely defined the instant of follow through they have chosen to properly compare with their data.

\section{Conclusions}

The weight transfer measured either using the reaction forces, the center of pressure of each foot or the total center of pressure, has been widely studied in the literature and is very useful for golf coaches and players. The reaction forces come from the interaction between the foot, the shoe, and the ground. However, until now there was not any quantitative 
information about the foot pressure distribution in players of different handicaps. We have demonstrated the existence of a statistically significant and consistent pattern of normalized foot pressure distribution, being identical for swings performed both using the driver and the 5-iron. We have also observed that there are significant differences between the pattern of professionals and amateur players. The obtained pattern agrees with the principle of weight transfer, meaning that at the lateral zones the pressure on the rear foot increases during the backswing and decreases during the downswing, while the lead foot has an inverse behavior. However, the medial parts of the foot make a reverse pressure pattern in comparison with the lateral areas, except for the medial arch and medial heel, which do not show this rear to lead foot transmission of load. Further studies are needed to confirm this pattern as a reference model, analyzing professional and amateur players using a larger set of clubs, including the driver, irons, woods, wedges, and putter. Indeed, our study has several limitations, such as a low sampling frequency, a limited number of professional players, lack of information about the horizontal forces, and lack of information about the medial and lateral areas of the toes. In future studies we intend to address these issues to confirm the attained results and gain further insights on the pressure pattern evolution of players with different handicaps.

Author Contributions: Conceptualization and methology (E.N., J.M.M. and D.L.); software (D.L., M.d.O.); formal analysis (E.N., D.L.); investigation (E.N., J.M.M.); resources, (S.F., E.N.); data curation (J.M.M.; M.d.O.); writing—original draft preparation (E.N., J.M.M., D.L., M.d.O.); writing一review and editing (E.N., J.M.M., S.F., D.L.); visualization, supervision, project administration, (S.F., E.N.); funding acquisition, (S.F., E.N.). All authors have read and agreed to the published version of the manuscript.

Funding: This research was funded by [Continental AG] grant number [\#P2111600007].

Institutional Review Board Statement: The study was conducted in accordance with the Declaration of Helsinki, and approved by the Ethics Committee of the Universidad Politécnica de Madrid (\#P2111600007; 22 November 2020).

Informed Consent Statement: Informed consent was obtained from all subjects involved in the study.

Data Availability Statement: All graphs of pressures of the foot areas during the swing can be required to the corresponding author (enrique.navarro@upm.es).

Acknowledgments: This work was funded by the Continental AG. The authors gratefully acknowledge the technical supports provided from Continental.

Conflicts of Interest: The authors declare no conflict of interest.

\section{References}

1. Nelson, L. A Better Weight Shift Made the Difference. Golf Dig. 1980, 37-38.

2. Leadbetter, D. A Lesson with Leadbetter: The Swing; Telstar Video Entertainment: London, UK, 1995; pp. $13-85$.

3. Leadbetter, D. Faults and Fixes: The Golfer's Essential Problem-Solving Manual; Collins Willow: London, UK, 1993.

4. Norman, G. Advanced Golf; Heinemann: Port Melbourne, Australia, 1995; pp. 150-153.

5. Barrentine, S.W.; Fleisig, G.S.; Johnson, H.; Woolley, T.W. Ground reaction forces and torques of professional and amateur golfers. In Science and golf II. Proceedings of the World Scientific Congress of Golf; Cochran, A.J., Farrally, M.R., Eds.; E and FN Spon: London, UK, 1994; pp. 33-39.

6. Chu, Y.; Sell, T.C.; Lephart, S.M. The relationship between biomechanical variables and driving performance during the golf swing. J. Sports Sci. 2010, 28, 1251-1259. [CrossRef] [PubMed]

7. McNitt-Gray, J.L.; Munaretto, J.; Zaferiou, A.; Requejo, P.S.; Flashner, H. Regulation of reaction forces during the golf swing. Sports Biomech. 2013, 12, 121-131. [CrossRef] [PubMed]

8. Choi, A.; Sim, T.; Mun, J.H. Improved determination of dynamic balance using the centre of mass and centre of pressure inclination variables in a complete golf swing cycle. J. Sports Sci. 2016, 34, 906-914. [CrossRef] [PubMed]

9. Han, K.H.; Como, C.; Kim, J.; Lee, S.; Kim, J.; Kim, D.K.; Kwon, Y. Effects of the golfer-ground interaction on clubhead speed in skilled male golfers. Sports Biomech. 2019, 18, 115-134. [CrossRef] [PubMed]

10. Williams, K.R.; Cavanagh, P.R.; Miller, J.D.; Parra, M.E.; Dimmick, H.L.; Herda, T.J. The mechanics of foot action during the golf swing and implications for shoe design. Med. Sci. Sports Exerc. 1983, 15, 247-255. [CrossRef] [PubMed] 
11. Koenig, G.; Tamres, M.; Mann, R.W. An analysis of the kinetics and kinematics of the golf swing. In Proceedings of the 11 International Symposium on Biomechanics in Sports, Amherst, MA, USA, 23-26 June 1993; pp. 328-333.

12. Ball, K.; Best, R. Different centre of pressure patterns within the golf stroke I: Cluster analysis. J. Sports Sci. 2007, 25, 757-770. [CrossRef] [PubMed]

13. Ball, K.; Best, R. Different centre of pressure patterns within the golf stroke II: Group-based analysis. J. Sports Sci. 2007, 25, 771-779. [CrossRef] [PubMed]

14. Ball, K.; Best, R. Golf styles and centre of pressure patterns when using different golf clubs. J. Sports Sci. 2011, 29, 587-590. [CrossRef] [PubMed]

15. Wallace, E.S.; Grimshaw, P.N.; Ashford, R.L. Discrete pressure profiles of the feet and weight transfer patterns during the golf swing. In Science and Golf II; Taylor \& Francis: Abingdon, UK, 1994; pp. $26-32$.

16. Kawashima, K.; Meshizuka, T.; Takeshita, S. A Kinematic Analysis of Foot Force Exerted on the Soles During the Golf Swing Among Skilled and Unskilled Golfers. In Science and Golf III, Proceedings of the World Scientific Congress of Golf; Taylor \& Francis: London, UK, 1999; pp. 40-45. Available online: https://www.tib.eu/de/suchen/id/BLCP\%3ACN028918786 (accessed on 10 September 2021).

17. Lee, E.; Kim, J.; Kim, D. Golf Swing Recognition Using Low-Cost Smart Insole. In Advances in Computer Science and Ubiquitous Computing; Springer: Singapore, 2019; pp. 396-400.

18. Pataky, T.C. Correlation between maximum in-shoe plantar pressures and clubhead speed in amateur golfers. J. Sports Sci. 2015, 33, 192-197. [CrossRef] [PubMed]

19. Worsfold, P.; Smith, N.A.; Dyson, R.J. Kinetic assessment of golf shoe outer sole design features. J. Sports Sci. Med. 2009, 8, 607-615. [PubMed]

20. Ball, K.; Best, R. Centre of pressure patterns in the golf swing: Individual-based analysis. Sports Biomech. 2012, 11, 175-189. [CrossRef] [PubMed]

21. Worsfold, P.; Smith, N.A.; Dyson, R.J. Low handicap golfers generate more torque at the shoe-natural grass interface when using a driver. J. Sports Sci. Med. 2008, 7, 408-414. Available online: https://www.ncbi.nlm.nih.gov/pubmed/24149910 (accessed on 10 September 2021). [PubMed]

22. Murakami, E.A.Y.; Mochimaru, M. Foot Pressure, Ground Reaction Force and 3D Motion Analysis of Golf Swing Applied to Spikeless Golf Shoe Development. In Proceedings of the 6th World Congress of Biomechanics (WCB 2010), Singapore, 1-6 August 2010; Volume 31, pp. 194-197. [CrossRef]

23. Luo, Z.P.; Berglund, L.J.; An, K.N. Validation of F-Scan pressure sensor system: A technical note. J. Rehabil. Res. Dev. 1998, 35, 186-191. [CrossRef] [PubMed]

24. Kwon, Y.; Como, C.S.; Singhal, K.; Lee, S.; Han, K.H. Assessment of planarity of the golf swing based on the functional swing plane of the clubhead and motion planes of the body points. Sport Biomech 2012, 11, 127-148. [CrossRef] [PubMed]

25. Hogan, B.; Wind, H.W. Ben Hogan's Five Lessons: The Modern Fundamentals of Golf/Five Lessons: The Modern Fundamentals of Golf; Barnes: New York, NY, USA, 1957. 